\title{
A new algorithm for computing the 2-dimensional matching distance between size functions
}

\author{
Silvia Biasotti ${ }^{\mathrm{c}}$, Andrea Cerri ${ }^{\mathrm{a}, \mathrm{b}}$, Patrizio Frosini ${ }^{\mathrm{a}, \mathrm{b}}$, Daniela Giorgi ${ }^{\mathrm{c}}$ \\ ${ }^{a}$ ARCES, Università di Bologna, via Toffano 2/2, I-40135 Bologna, Italia \\ ${ }^{b}$ Dipartimento di Matematica, Università di Bologna, P.zza di Porta S. Donato 5, I-40126 Bologna, Italia \\ ${ }^{c}$ Istituto di Matematica Applicata e Tecnologie Informatiche, Consiglio Nazionale delle Ricerche Via De Marini 6, I-16149 Genova, Italy
}

\begin{abstract}
Size Theory has proven to be a useful geometrical/topological approach to shape analysis and comparison. Originally introduced by considering 1-dimensional properties of shapes, described by means of real-valued functions, it has been subsequently generalized to take into account multidimensional properties coded by functions valued in $\mathbb{R}^{k}$. In the context of Size Theory, this generalization has led to introduce a shape descriptor called $k$-dimensional size function, and a distance to compare size functions, namely the $k$-dimensional matching distance.

This paper proposes a novel computational framework to deal with the 2-dimensional case of Size Theory. More precisely, some new theoretical results about approximating the 2-dimensional matching distance are presented, leading to the formulation of an algorithm for its computation (up to an arbitrary error threshold).

Keywords: multidimensional size function, Size Theory, shape comparison
\end{abstract}

\section{Introduction}

Shape Comparison and Retrieval are challenging issues in Computer Vision, Computer Graphics, Image Analysis and Pattern Recognition. Shape models, including 2D images and 3D objects, possess a considerable amount of visual and semantic information that, to be fully exploited, needs the definition of powerful description, classification and retrieval techniques [22, 23]. Recently, Persistent Topology - including Size Theory and Persistent Homology - has considerably grown in popularity and has been proven to provide both theoretical and computational tools for shape comparison.

The main idea behind this research field is to take into account topology shape features with respect to some geometric properties conveyed by real functions defined on the shape itself $[3,9,16]$. In formal settings, that means that a shape is represented by a pair $(X, \varphi)$, where $X$ is a topological space and $\varphi: X \rightarrow \mathbb{R}$ is a continuous real-valued function called measuring (or filtering) function. Size Theory was introduced in the early 90's to allow one to store quantitatively some qualitative information about $(X, \varphi)$. In particular, its size function $\ell_{(X, \varphi)}$ is a shape descriptor

Email addresses: silvia@ge.imati.cnr.it (Silvia Biasotti), cerri@dm. unibo.it (Andrea Cerri), frosini@dm.unibo.it (Patrizio Frosini), daniela@ge.imati.cnr.it (Daniela Giorgi) 
encoding the evolution of the 0-th Betti number in the sublevel sets of $X$ induced by $\varphi$. Size functions are complete and stable descriptors, admitting a simple and compact representation made up of a multiset of points in the Euclidean plane, and are compared using a suitable matching distance [3].

In their original formulation, size functions have been widely studied and applied to Pattern Recognition problems $[4,7,13,24,25]$. Over the years, similar ideas have been re-proposed by Persistent Homology according to a homological approach and have found applications in shape description and data simplification [14, 15].

Considering the general scenario of shape analysis, a single real-valued measuring function is not enough to cope with the shape description problem. In fact, data are often characterized by two or more properties; this happens for example with physical simulations, where several measurements are made about an observed phenomenon, or when data have multidimensional features, such as colors in the RGB model. These considerations have recently drawn the attention to the study of a multidimensional setting $[1,2,6,14,19]$. The term multidimensional, or equivalently $k$ dimensional, is related to considering measuring functions taking value in $\mathbb{R}^{k}$, that is, $\vec{\varphi}: X \rightarrow \mathbb{R}^{k}$, and the subsequent extension of shape descriptors to this case.

Despite the need of managing multi-dimensional data, not so much has been done from the point of view of applications. This is due to the fact that a complete, discrete and stable representation for the Persistent Topology shape descriptors seems not to be available in the multidimensional setting, differently from what happens in the 1dimensional situation. The arising computational difficulties have been faced following different strategies $[1,5,10]$, but not completely solved.

As a partial solution, in [1] the authors studied the concept of $k$-dimensional size functions and proved that the restrictions of a $k$-dimensional size function to suitable subsets of its domain turn out to be 1-dimensional. This allowed the definition of a stable matching distance between $k$-dimensional size functions, namely the $k$-dimensional matching distance, building on existing results for the 1-dimensional case. Unfortunately, [1] does not explain how to approximate the matching distance in a way to obtain a good compromise between computational cost and quality of results. Indeed, the straightforward application of the method could require a very huge number of calculations (see also [2]).

This paper yields a theoretical and computational solution of the problem when $k=2$. The theoretical results proven in Lemma 3.1, Lemma 3.3 and Theorem 3.4 allow us to bound the computational error in evaluating the matching distance between 2-dimensional size functions. On these bases we develop an algorithm to approximate the 2-dimensional matching distance up to an arbitrary error threshold, which represents the maximum admissible error. Experimental results on 3D objects represented by surface meshes demonstrate the efficiency of the algorithm to reduce the number of calculations required to approximate the matching distance.

The remainder of the paper is organized as follows. In Section 2 we overview main definitions and properties about size functions; the study of the 1-dimensional case (Section 2.1) is necessary as it provides the basis for the definition of a complete representation for size functions in the 2-dimensional case (Section 2.2). Our new results are given in Section 3, along with the novel algorithm we propose (Section 3.2). Then, experiments on 3D models 


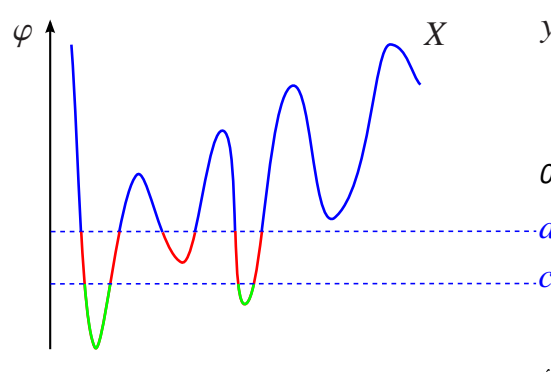

(A)

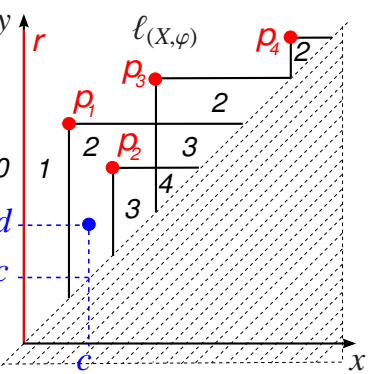

(E)

Figure 1: (a) The topological space $X$ and the measuring function $\varphi$. (b) The associated size function $\ell_{(X, \varphi)}$. To compute the value of $\ell_{(X, \varphi)}$ at the point $(c, d)$, it is sufficient to count how many of the three connected components in the sublevel $X\langle\varphi \leq d\rangle$ contain at least one point of $X\langle\varphi \leq c\rangle$ : it can be easily checked that $\ell_{(X, \varphi)}(c, d)=2$.

(Section 4) are shown to validate our proposal. Some discussions in Section 5 conclude the paper.

\section{Preliminary results}

In this section we review some basic concepts that have been introduced in Size Theory. For further details the reader is referred to [3].

\subsection{1-dimensional size functions}

Size functions are shape descriptors that code the topological evolution of the sublevel sets of a space $X$, according to the increasing values of a real function $\varphi: X \rightarrow \mathbb{R}$ defined on it; $\varphi$ is called 1-dimensional measuring function. Indeed, size functions count the number of connected components which remain disconnected passing from a lower level set of $X, X_{u}=\{P \in X: \varphi(P) \leq u\}$, to another. Since the sequence of lower level sets is driven by the real function $\varphi$, size functions encode the geometrical properties of $X$ captured by $\varphi$ in the topological evolution of $X_{u}$. More formally,

Definition 2.1. Given a size pair $(X, \varphi)$ with $X$ a non-empty, compact and locally connected Hausdorff space and $\varphi$ a continuous function, and denoting $\Delta^{+}=\{(u, v) \in \mathbb{R} \times \mathbb{R}: u<v\}$, the size function of $(X, \varphi)$ is $\ell_{(X, \varphi)}: \Delta^{+} \rightarrow \mathbb{N}$ with $\ell_{(X, \varphi)}(u, v)$ equal to the number of connected components of the lower level set $X_{v}=\{P \in X: \varphi(P) \leq v\}$, containing at least one point of the lower level set $X_{u}$.

Figure 1 shows an example of a size pair $(X, \varphi)$ together with the size function $\ell_{(X, \varphi)}$. Figure $1(a)$ shows the size pair $(X, \varphi)$, where $X$ is the curve drawn by a solid line, and $\varphi$ is the ordinate function. Figure $1(b)$ shows the associated 1-dimensional size function $\ell_{(X, \varphi)}$. The domain $\Delta^{+}=\left\{(u, v) \in \mathbb{R}^{2}: u<v\right\}$ is divided into regions. Each one is labeled by a number, coinciding with the constant value that $\ell_{(X, \varphi)}$ takes in the interior of that region.

Roughly speaking, each 1-dimensional size function can be seen as a linear combination (with natural numbers as coefficients) of characteristic functions associated to the (possibly unbounded) triangles laying on the domain $\Delta^{+}$ 
[17]. The bounded triangles are of the form $\left\{(u, v) \in \Delta^{+}: \alpha \leq u<v<\beta\right\}$, while the unbounded ones are of the form $\left\{(u, v) \in \Delta^{+}: \eta \leq u<v\right\}$. Hence, a simple and compact representation is obtained by associating the set $\left\{(u, v) \in \Delta^{+}: \alpha \leq u<v<\beta\right\}$ to the point $(\alpha, \beta)$, and the set $\left\{(u, v) \in \Delta^{+}: \eta \leq u<v\right\}$ to the point at infinity $(\eta, \infty)$. The points of a formal series having finite coordinates are called proper cornerpoints, while the ones with a coordinate at infinity are said to be cornerpoints at infinity or cornerlines. For example, the size function $\ell_{(X, \varphi)}$ shown in Figure $1(b)$ admits the representation by formal series given by $r+p_{1}+p_{2}+p_{3}+p_{4}$, where $r$ is the only cornerpoint at infinity, with coordinates $(0, \infty)[17]$.

The combinatorial representation of size functions using cornerpoints implies that size functions can be compared via a suitable distance between formal series, namely the matching distance, see details in [12]. Roughly speaking, the matching distance $d_{\text {match }}$ can be seen as a measure of the cost of transporting the cornerpoints of a 1-dimensional size function into the cornerpoints of another one.

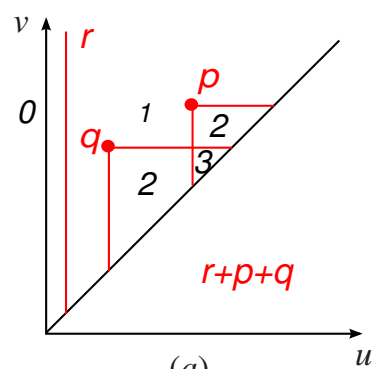

(a)

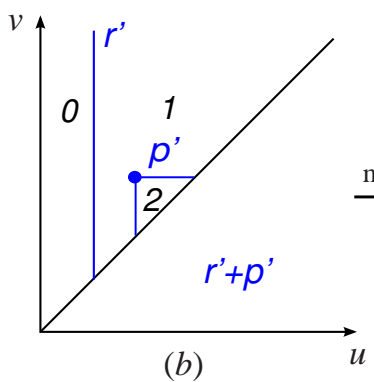

(b)
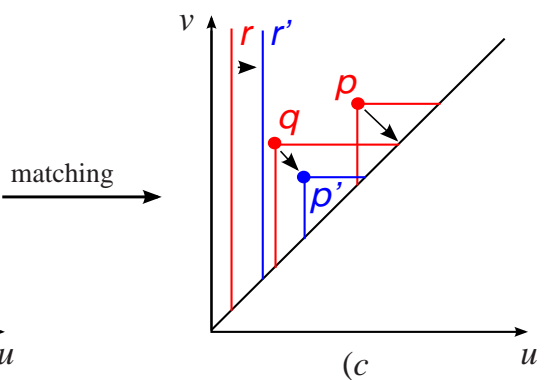

Figure 2: (a) The size function corresponding to the formal series $r+p+q$. (b) The size function corresponding to the formal series $r^{\prime}+p^{\prime}$. (c) The matching between the two formal series, realizing the matching distance between the two size functions.

Formally, let us consider the 1-dimensional size functions $\ell_{1}, \ell_{2}$ and their multiset $C_{1}$ (respectively $C_{2}$ ) of cornerpoints for $\ell_{1}$ (resp. $\ell_{2}$ ), counted with their multiplicities and augmented by adding the points of the diagonal $\left\{(u, v) \in \mathbb{R}^{2}: u=v\right\}$ counted with infinite multiplicity. If we denote by $\overline{\Delta^{*}}$ the set $\overline{\Delta^{+}}$extended by the points at infinity of the kind $(a, \infty)$, i.e. $\overline{\Delta^{*}}=\overline{\Delta^{+}} \cup\{(a, \infty): a \in \mathbb{R}\}$, the matching distance $d_{\text {match }}\left(\ell_{1}, \ell_{2}\right)$ is then defined as

$$
d_{\text {match }}\left(\ell_{1}, \ell_{2}\right)=\min _{\sigma} \max _{P \in C_{1}} \delta(P, \sigma(P))
$$

where $\sigma$ varies among all the bijections between $C_{1}$ and $C_{2}$ and

$$
\delta\left((u, v),\left(u^{\prime}, v^{\prime}\right)\right)=\min \left\{\max \left\{\left|u-u^{\prime}\right|,\left|v-v^{\prime}\right|\right\}, \max \left\{\frac{v-u}{2}, \frac{v^{\prime}-u^{\prime}}{2}\right\}\right\},
$$

for every $(u, v),\left(u^{\prime}, v^{\prime}\right) \in \overline{\Delta^{*}}$, with the convention about $\infty$ that $\infty-v=v-\infty=\infty$ when $v \neq \infty, \infty-\infty=0, \frac{\infty}{2}=\infty$, $|\infty|=\infty, \min \{c, \infty\}=c$ and $\max \{c, \infty\}=\infty$.

The pseudometric ${ }^{1} \delta$ measures the pseudo-distance between two points $(u, v)$ and $\left(u^{\prime}, v^{\prime}\right)$ as the minimum between

\footnotetext{
${ }^{1}$ A pseudo-distance $d$ is just a distance missing the condition $d(X, Y)=0 \Rightarrow X=Y$, i.e. two distinct elements may have vanishing distance with respect to $d$.
} 
the cost of moving one point onto the other and the cost of moving both points onto the diagonal, with respect to the max-norm and under the assumption that any two points of the diagonal have vanishing pseudo-distance. As different 1-dimensional size functions may in general have a different number of cornerpoints, $d_{\text {match }}$ allows a proper cornerpoint to be matched to a point of the diagonal: This matching can be interpreted as the deletion of a proper cornerpoint, see Figure 2(c).

The matching distance is stable with respect to perturbations of the measuring functions. Indeed, the following Matching Stability Theorem has been proven [11, 12] (see also [9]):

Theorem 2.2 (Matching Stability Theorem). If $(X, \varphi),(Y, \psi)$ are two 1-dimensional size pairs with $X, Y$ homeomorphic, then $d_{\text {match }}\left(\ell_{(X, \varphi)}, \ell_{(Y, \psi)}\right) \leq \inf _{h: X \rightarrow Y} \max _{x \in X}|\varphi(x)-\psi(h(x))|$, where $h$ varies among all possible homeomorphisms from $X$ to $Y$.

Remark 2.3. The hypothesis that $X$ and $Y$ are homeomorphic is not so restrictive. Indeed, in [18] it has been proven that, in most of applicative contexts of pattern recognition, the use of the multidimensional setting allows us to substitute $X$ and $Y$ with a unique compact set $K \subset \mathbb{R}^{m}$.

\subsection{2-dimensional size functions}

Let $X$ be a non-empty, compact and locally connected Hausdorff space, as in the 1-dimensional case, and $\vec{\varphi}=$ $\left(\varphi_{1}, \varphi_{2}\right): X \rightarrow \mathbb{R}^{2}$ be a continuous function, called 2-dimensional measuring function. The following relations $\leq$ and $<$ are considered in $\mathbb{R}^{2}$ : for $\vec{u}=\left(u_{1}, u_{2}\right)$ and $\vec{v}=\left(v_{1}, v_{2}\right), \vec{u} \leq \vec{v}$ (resp. $\vec{u}<\vec{v}$ ) if and only if $u_{i} \leq v_{i}$ (resp. $\left.u_{i}<v_{i}\right)$ for $i=1$,2. Moreover, we set $\|\vec{u}\|_{\infty}=\max _{i=1,2}\left|u_{i}\right|$. For every $\vec{u}, \vec{v} \in \mathbb{R}^{2}$, the lower level set is defined as $X\langle\vec{\varphi} \leq \vec{u}\rangle=\left\{x \in X: \varphi_{i}(x) \leq u_{i}, i=1,2\right\}$, whereas we denote $\Delta^{+}=\left\{(\vec{u}, \vec{v}) \in \mathbb{R}^{2} \times \mathbb{R}^{2}: \vec{u}<\vec{v}\right\}$.

Extending the definition of size functions to the 2-dimensional case is straightforward:

Definition 2.4. The (2-dimensional) size function of the size pair $(X, \vec{\varphi})$ is the function $\ell_{(X, \vec{\varphi})}: \Delta^{+} \rightarrow \mathbb{N}$, defined by setting $\ell_{(X, \vec{\varphi})}(\vec{u}, \vec{v})$ equal to the number of connected components in the set $X\langle\vec{\varphi} \leq \vec{v}\rangle$ containing at least one point of $X\langle\vec{\varphi} \leq \vec{u}\rangle$.

To combinatorially represent 2-dimensional size functions in terms of cornerpoints as in the 1-dimensional case is not straightforward, and consequently to define a stable and computable distance between 2-dimensional size functions is not trivial.

The rest of this Section shows how the framework of 2-dimensional size functions can be reduced to the case $k=1$, by a change of variable and the use of a suitable foliation, using a strategy similar to the one in [1]. The main idea is to provide a parameterized family of half-planes in $\mathbb{R}^{2} \times \mathbb{R}^{2}$, and prove that the restriction of a 2-dimensional size function $\ell_{(X, \vec{\varphi})}$ to each of these half-planes turns out to be a particular 1-dimensional size function. This implies that we can build on the results proved in the 1-dimensional case to develop the theory in the 2-dimensional case. 
First of all, let us define the collection of half-planes foliating $\Delta^{+}$. The foliation we introduce here slightly differs from the one given in [1, Def. 7] and corresponds to a re-parameterization of the half-planes that does not affect the stability results (see [8] for details).

Definition 2.5. A pair $(\vec{\lambda}, \vec{\beta})$, with $\vec{\lambda}=\left(\lambda_{1}, \lambda_{2}\right) \in \mathbb{R}^{2}$ and $\vec{\beta}=\left(\beta_{1}, \beta_{2}\right) \in \mathbb{R}^{2}$, is linearly admissible if: (a) $\lambda_{1}, \lambda_{2}>0$; (b) $\lambda_{1}+\lambda_{2}=1$; (c) $\beta_{1}+\beta_{2}=0$. Denoting by $\operatorname{Ladm}_{2}$ the set of all linearly admissible pairs in $\mathbb{R}^{2} \times \mathbb{R}^{2}$, for every $(\vec{\lambda}, \vec{\beta}) \in \operatorname{Ladm}_{2}$, let us define the half-plane $\pi_{(\vec{\lambda}, \vec{\beta})}$ of $\mathbb{R}^{2} \times \mathbb{R}^{2}$ by the parametric equations $\vec{u}=\sigma \vec{\lambda}+\vec{\beta}, \vec{v}=\tau \vec{\lambda}+\vec{\beta}$, with $\sigma, \tau \in \mathbb{R}$ and $\sigma<\tau$.

The next proposition shows the main properties of the collection $\left\{\pi_{(\vec{\lambda}, \vec{\beta})}\right\}_{(\vec{\lambda}, \vec{\beta}) \in \operatorname{Ladm}_{2}}$.

Proposition 2.6. For every $(\vec{u}, \vec{v}) \in \Delta^{+}$there exists one and only one linearly admissible pair $(\vec{\lambda}, \vec{\beta})$ such that $(\vec{u}, \vec{v}) \in$ $\pi_{(\vec{\lambda}, \vec{\beta})}$. Moreover, for every $(\vec{\lambda}, \vec{\beta}) \in$ Ladm $_{2}$, the half-plane $\pi_{(\vec{\lambda}, \vec{\beta})}$ is contained in $\Delta^{+}$.

Proof. If $(\vec{u}, \vec{v}) \in \Delta^{+}$and $(\vec{\lambda}, \vec{\beta}) \in \operatorname{Ladm}_{2}$, it can be easily verified that $\vec{u}=\sigma \vec{\lambda}+\vec{\beta}, \vec{v}=\tau \vec{\lambda}+\vec{\beta}$ if and only if, for $i=1,2$,

$$
\lambda_{i}=\frac{v_{i}-u_{i}}{\sum_{j=1}^{2}\left(v_{j}-u_{j}\right)}, \quad \beta_{i}=\frac{u_{i} \sum_{j=1}^{2} v_{j}-v_{i} \sum_{j=1}^{2} u_{j}}{\sum_{j=1}^{2}\left(v_{j}-u_{j}\right)}, \quad \sigma=u_{1}+u_{2}, \quad \tau=v_{1}+v_{2} .
$$

On the other hand, it is trivial to check that each half-plane $\pi_{(\vec{\lambda}, \vec{\beta})}: \vec{u}=\sigma \vec{\lambda}+\vec{\beta}, \vec{v}=\tau \vec{\lambda}+\vec{\beta}$, with $\sigma, \tau \in \mathbb{R}$ and $\sigma<\tau$, is contained in $\Delta^{+}$provided that $(\vec{\lambda}, \vec{\beta}) \in \operatorname{Ladm}_{2}$.

On the basis of Proposition 2.6 it is possible to reduce the 2-dimensional setting to the case $k=1$.

Theorem 2.7 (Reduction Theorem). Let $(\vec{\lambda}, \vec{\beta}) \in \operatorname{Ladm}_{2}$, and let $F_{(\vec{\lambda}, \vec{\beta})}^{\vec{\phi}}: X \rightarrow \mathbb{R}$ be the function defined by setting

$$
F_{(\vec{\lambda}, \vec{\beta})}^{\vec{\varphi}}(x)=\max \left\{\frac{\varphi_{1}(x)-\beta_{1}}{\lambda_{1}}, \frac{\varphi_{2}(x)-\beta_{2}}{\lambda_{2}}\right\} .
$$

Then, for every $(\vec{u}, \vec{v})=(\sigma \vec{\lambda}+\vec{\beta}, \tau \vec{\lambda}+\vec{\beta}) \in \pi_{(\vec{\lambda}, \vec{\beta})}$ it holds that $\ell_{(X, \vec{\varphi})}(\vec{u}, \vec{v})=\ell_{\left(X, F_{(\vec{\lambda}, \vec{\beta})}^{\vec{\beta}}\right)}(\sigma, \tau)$.

The proof of Theorem 2.7 can be straightforwardly derived from the proof of [1, Thm. 3].

Roughly speaking, the Reduction Theorem 2.7 states that, on each half-plane of the collection $\left\{\pi_{(\vec{\lambda}, \vec{\beta})}\right\}_{(\vec{\lambda}, \vec{\beta}) \in \operatorname{Ladm} m_{2}}$, the restriction of a given 2-dimensional size function coincides with a particular size function in two scalar variables, i.e. a 1-dimensional one. A first important consequence is the possibility of representing a 2-dimensional size function $\ell_{(X, \vec{\varphi})}$ by a collection of formal series of points and lines, following the machinery described in Subsection 2.1 for the case $k=1$. Therefore, the matching distance between 1-dimensional size functions can be applied to every halfplane of the foliation $\left\{\pi_{(\vec{\lambda}, \vec{\beta})}\right\}_{(\vec{\lambda}, \vec{\beta}) \in \operatorname{Ladm}_{2}}$, showing that it is stable with respect to perturbations of the multidimensional measuring functions and to the choice of the leaves of the foliation (cf. [1, Propositions 2 and 3]). These stability properties lead to the following definition of a distance between 2-dimensional size functions ${ }^{2}$ (see also [1, Def. 8]).

\footnotetext{
${ }^{2}[8]$ has proven that the distance in Definition 2.8 coincides with the restriction to the 2-dimensional case of the one given in [1, Def. 8], which is stable.
} 
Definition 2.8. Let $(X, \vec{\varphi})$ and $(Y, \vec{\psi})$ be two size pairs, with $\vec{\varphi}, \vec{\psi}$ valued in $\mathbb{R}^{2}$. The 2-dimensional matching distance $D_{\text {match }}\left(\ell_{(X, \vec{\varphi})}, \ell_{(Y, \vec{\psi})}\right)$ is the (extended $\left.{ }^{3}\right)$ distance defined by setting

$$
D_{\text {match }}\left(\ell_{(X, \vec{\varphi})}, \ell_{(Y, \vec{\psi})}\right)=\sup _{(\vec{\lambda}, \vec{\beta})} d_{\left(\vec{A} a d m_{2}\right.} d_{(\vec{\lambda}, \vec{\beta})}\left(\ell_{(X, \vec{\varphi})}, \ell_{(Y, \vec{\psi})}\right),
$$

where

$$
\left.d_{(\vec{\lambda}, \vec{\beta})}\left(\ell_{(X, \vec{\varphi})}, \ell_{(Y, \vec{\psi})}\right)=\min _{i=1,2} \lambda_{i} \cdot d_{\text {match }}\left(\ell_{\left(X, F_{(\vec{\lambda}, \vec{\beta}}^{\vec{\phi}}\right)}, \ell_{\left(Y, F_{(}^{\vec{\psi}} \overrightarrow{\vec{\beta}}\right)}\right)\right) .
$$

Taking a non-empty and finite subset $A \subseteq \operatorname{Ladm}_{2}$ and replacing $\sup _{(\vec{\lambda}, \vec{\beta}) \in \operatorname{Ladm} 2}$ by $\max _{(\vec{\lambda}, \vec{\beta}) \in A}$ in Definition 2.8, we obtain a stable and computable pseudo-distance between 2-dimensional size functions.

\section{Efficient computation of the 2-dimensional matching distance}

This Section describes a method able to automatically approximate the 2-dimensional matching distance $D_{\text {match }}$ between two 2-dimensional size functions $\ell_{(X, \vec{\varphi})}$ and $\ell_{(Y, \vec{\psi})}$ up to an error threshold $\varepsilon$. Using the notation introduced in Section 2.2, this means to opportunely define the subset $A \subseteq L a d m_{2}$ so as to reach a compromise between computational cost and quality of approximation.

\subsection{New approximation results}

Given the threshold value $\varepsilon$, our algorithm automatically provides as output the distance $\widetilde{D}_{\text {match }}\left(\ell_{(X, \vec{\varphi})}, \ell_{(Y, \vec{\psi})}\right)$ such that $\left|D_{\text {match }}\left(\ell_{(X, \vec{\varphi})}, \ell_{(Y, \vec{\psi})}\right)-\widetilde{D}_{\text {match }}\left(\ell_{(X, \vec{\varphi})}, \ell_{(Y, \vec{\psi})}\right)\right| \leq \varepsilon$

Let us consider the 2-dimensional size pairs $(X, \vec{\varphi})$ and $(Y, \vec{\psi})$, and assume that $X$ and $Y$ are homeomorphic, so that $D_{\text {match }}\left(\ell_{(X, \vec{\psi})}, \ell_{(Y, \vec{\psi})}\right)<\infty$ (this is a not so restrictive requirement as discussed in Remark 2.3). The first step toward the formulation of our algorithm is to estimate the changing of $d_{(\vec{\lambda}, \vec{\beta})}\left(\ell_{(X, \vec{\psi})}, \ell_{(Y, \vec{\psi})}\right)$, the pair $(\vec{\lambda}, \vec{\beta})$ varying in $\operatorname{Ladm}_{2}$, i.e. when moving from one leaf to another in the half-plane foliation of $\Delta^{+}$.

We start by observing that $\operatorname{Ladm}_{2}=\left\{(\vec{\lambda}, \vec{\beta})=\left(\lambda_{1}, \lambda_{2}, \beta_{1}, \beta_{2}\right) \in \mathbb{R}^{2} \times \mathbb{R}^{2}: \lambda_{1}+\lambda_{2}=1, \beta_{1}+\beta_{2}=0,0<\lambda_{1}<1\right\}=$ $\left\{(a, 1-a, b,-b) \in \mathbb{R}^{2} \times \mathbb{R}^{2}: 0<a<1\right\}$. In what follows, for every $\vec{\lambda}=(a, 1-a)$ with $a \in(0,1)$, we shall denote the value $\min \{a, 1-a\}$ by $\mu(\vec{\lambda})$.

The next Lemma 3.1 claims that the evaluation of $d_{(\vec{\lambda}, \vec{\beta})}\left(\ell_{(X, \vec{\varphi})}, \ell_{(Y, \vec{\psi})}\right)$ can be avoided in a large part of $\operatorname{Ladm}_{2}$. Fix $C=\max \left\{\max _{x \in X}\|\vec{\varphi}(x)\|_{\infty}, \max _{y \in Y}\|\vec{\psi}(y)\|_{\infty}\right\}$, and consider the set

$$
\operatorname{Ladm}_{2}^{*}=\left\{(a, 1-a, b,-b) \in \operatorname{Ladm}_{2}:|b|<C\right\} .
$$

\footnotetext{
${ }^{3}$ The term "extended" refers to the fact that, if the spaces $X$ and $Y$ are not assumed to be homeomorphic, the multidimensional matching distance $D_{\text {match }}$ still verifies all the properties of a distance, except for the fact that it may take the value $\infty$
} 
Lemma 3.1. Let $(\vec{\lambda}, \vec{\beta}) \in \operatorname{Ladm}_{2} \backslash \operatorname{Ladm}_{2}^{*}$, with $(\vec{\lambda}, \vec{\beta})=(a, 1-a, b,-b)$. Then it follows that

$$
d_{(\vec{\lambda}, \vec{b})}\left(\ell_{(X, \vec{\varphi})}, \ell_{(Y, \vec{\psi})}\right)= \begin{cases}\frac{\mu(\vec{\lambda})}{a} \cdot d_{\text {match }}\left(\ell_{\left(X, \varphi_{1}\right)}, \ell_{\left(Y, \psi_{1}\right)}\right), & \text { if } b \leq-C ; \\ \frac{\mu(\vec{\lambda})}{1-a} \cdot d_{\text {match }}\left(\ell_{\left(X, \varphi_{2}\right)}, \ell_{\left(Y, \psi_{2}\right)}\right), & \text { if } b \geq C .\end{cases}
$$

Proof. It is sufficient to observe that $b \leq-C$ implies $F_{(\vec{\lambda}, \vec{\beta})}^{\vec{\varphi}}(x)=\frac{\varphi_{1}(x)-b}{a}$ for every $x \in X$, and $F_{(\vec{\lambda}, \vec{\beta})}^{\vec{\psi}}(y)=\frac{\psi_{1}(y)-b}{a}$ for every $y \in Y$, while $b \geq C$ implies $F_{(\vec{\lambda}, \vec{\beta})}^{\vec{\phi}}(x)=\frac{\varphi_{2}(x)+b}{1-a}$ for every $x \in X$ and $F_{(\vec{\lambda}, \vec{\beta})}^{\vec{\psi}}(y)=\frac{\psi_{2}(y)+b}{1-a}$ for every $y \in Y$. From the definition of the 1-dimensional matching distance $d_{\text {match }}$ (see also [8, Proposition 2.2]) the claim easily follows.

Remark 3.2. From Lemma 3.1 the maximum of $d_{(\vec{\lambda}, \vec{\beta})}\left(\ell_{(X, \vec{\varphi})}, \ell_{(Y, \vec{\psi})}\right)$ over $\left\{(\vec{\lambda}, \vec{b})=(a, 1-a, b,-b) \in \operatorname{Ladm}_{2}: b \leq-C\right\}$ is assumed when $a \leq \frac{1}{2}$, and it equals $d_{\text {match }}\left(\ell_{\left(X, \varphi_{1}\right)}, \ell_{\left(Y, \psi_{1}\right)}\right)$. Analogously, the maximum value for $d_{(\vec{\lambda}, \vec{\beta})}\left(\ell_{(X, \vec{\varphi})}, \ell_{(Y, \vec{\psi})}\right)$ over $\left\{(\vec{\lambda}, \vec{\beta}) \in \operatorname{Ladm}_{2}: b \geq C\right\}$ is assumed when $a \geq \frac{1}{2}$, and it equals $d_{\text {match }}\left(\ell_{\left(X, \varphi_{2}\right)}, \ell_{\left(Y, \psi_{2}\right)}\right)$.

According to Lemma 3.1 and Remark 3.2, in order to know the values $d_{(\vec{\lambda}, \vec{\beta})}\left(\ell_{(X, \vec{\varphi})}, \ell_{(Y, \vec{\psi})}\right)$ when $(\vec{\lambda}, \vec{\beta}) \in \operatorname{Ladm}_{2} \backslash$ $L a d m_{2}^{*}$, it is sufficient to consider just two suitable points of that region, e.g., the points whose coordinates are $\left(\frac{1}{2}, \frac{1}{2}, C+1,-(C+1)\right)$ and $\left(\frac{1}{2}, \frac{1}{2},-(C+1), C+1\right)$.

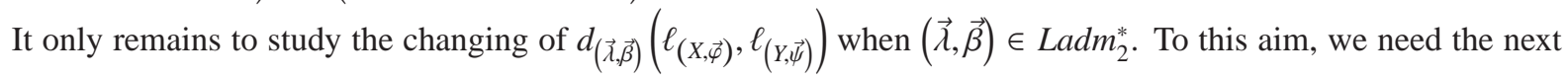
result.

Lemma 3.3. Assume $(\vec{\lambda}, \vec{\beta}) \in \operatorname{Ladm}_{2}^{*}$ and $\left(\vec{\lambda}^{\prime}, \vec{\beta}^{\prime}\right) \in \operatorname{Ladm}_{2}$, with $\left\|(\vec{\lambda}, \vec{\beta})-\left(\vec{\lambda}^{\prime}, \vec{\beta}^{\prime}\right)\right\|_{\infty} \leq \delta$. Then it follows that

$$
\left|d_{(\vec{\lambda}, \vec{\beta})}\left(\ell_{(X, \vec{\psi})}, \ell_{(Y, \vec{\psi})}\right)-d_{\left(\vec{x}^{\prime}, \vec{\beta}^{\prime}\right)}\left(\ell_{(X, \vec{\varphi})}, \ell_{(Y, \vec{\psi})}\right)\right| \leq \delta \cdot(16 C+2)
$$

Since the proof of Lemma 3.3 is rather technical, we prefer to confine it in Appendix A.

The previous Lemmas 3.1 and 3.3 can be merged together to obtain the following more general result.

Theorem 3.4 (Error Bound Theorem). If $(\vec{\lambda}, \vec{\beta}),\left(\vec{\lambda}^{\prime}, \vec{\beta}^{\prime}\right) \in \operatorname{Ladm}_{2}$, and $\left\|(\vec{\lambda}, \vec{\beta})-\left(\vec{\lambda}^{\prime}, \vec{\beta}^{\prime}\right)\right\|_{\infty} \leq \delta$, then it follows that

$$
\left|d_{(\vec{\lambda}, \vec{\beta})}\left(\ell_{(X, \vec{\psi})}, \ell_{(Y, \vec{\psi})}\right)-d_{\left(\vec{\lambda}^{\prime}, \vec{\beta}^{\prime}\right)}\left(\ell_{(X, \vec{\varphi})}, \ell_{(Y, \vec{\psi})}\right)\right| \leq \delta \cdot(16 C+2)
$$

Proof. Set $(\vec{\lambda}, \vec{\beta})=(a, 1-a, b,-b),\left(\vec{\lambda}^{\prime}, \vec{\beta}^{\prime}\right)=\left(a^{\prime}, 1-a^{\prime}, b^{\prime},-b^{\prime}\right)$. By Lemma 3.3, we only need to prove our statement when $(\vec{\lambda}, \vec{\beta}),\left(\vec{\lambda}^{\prime}, \vec{\beta}^{\prime}\right) \in \operatorname{Ladm}_{2} \backslash \operatorname{Ladm}_{2}^{*}$.

Let us first assume that $b, b^{\prime} \leq-C$. By Lemma 3.1, we have that

$$
\left|d_{(\vec{\lambda}, \vec{\beta})}\left(\ell_{(X, \vec{\varphi})}, \ell_{(Y, \vec{\psi})}\right)-d_{\left(\vec{\lambda}^{\prime}, \vec{\beta}^{\prime}\right)}\left(\ell_{(X, \vec{\varphi})}, \ell_{(Y, \vec{\psi})}\right)\right|=d_{\text {match }}\left(\ell_{\left(X, \varphi_{1}\right)}, \ell_{\left(Y, \psi_{1}\right)}\right)\left|\frac{\mu(\vec{\lambda})}{a}-\frac{\mu\left(\vec{\lambda}^{\prime}\right)}{a^{\prime}}\right| \leq 2 C\left|\frac{\mu(\vec{\lambda})}{a}-\frac{\mu\left(\vec{\lambda}^{\prime}\right)}{a^{\prime}}\right|,
$$

where the last inequality follows from Theorem 2.2.

If $\mu(\vec{\lambda})=a$ and $\mu\left(\vec{\lambda}^{\prime}\right)=a^{\prime}$, then it holds that $\frac{\mu(\vec{\lambda})}{a}=\frac{\mu\left(\vec{\lambda}^{\prime}\right)}{a^{\prime}}=1$. Therefore, in (1) we have $\left|\frac{\mu(\vec{\lambda})}{a}-\frac{\mu\left(\vec{\lambda}^{\prime}\right)}{a^{\prime}}\right|=0$, thus implying that $\left|d_{(\vec{\lambda}, \vec{\beta})}\left(\ell_{(X, \vec{\varphi})}, \ell_{(Y, \vec{\psi})}\right)-d_{\left(\vec{\lambda}^{\prime}, \vec{\beta}^{\prime}\right)}\left(\ell_{(X, \vec{\varphi})}, \ell_{(Y, \vec{\psi})}\right)\right|=0$. 


$$
\text { If } \mu(\vec{\lambda})=a \text { and } \mu\left(\vec{\lambda}^{\prime}\right)=1-a^{\prime} \text {, then } a \leq \frac{1}{2}, a^{\prime} \geq \frac{1}{2} \text { and we can write (observe that }\left|\mu(\vec{\lambda})-\mu\left(\vec{\lambda}^{\prime}\right)\right| \leq\left|a-a^{\prime}\right| \text { ) }
$$

$$
\begin{aligned}
\left|\frac{\mu(\vec{\lambda})}{a}-\frac{\mu\left(\vec{\lambda}^{\prime}\right)}{a^{\prime}}\right| & =\left|\frac{a^{\prime} \mu(\vec{\lambda})-a \mu\left(\vec{\lambda}^{\prime}\right)}{a a^{\prime}}\right|=\left|\frac{a^{\prime} \mu(\vec{\lambda})-a \mu(\vec{\lambda})+a \mu(\vec{\lambda})-a \mu\left(\vec{\lambda}^{\prime}\right)}{a a^{\prime}}\right|= \\
& =\left|\frac{\mu(\vec{\lambda})\left(a^{\prime}-a\right)+a\left(\mu(\vec{\lambda})-\mu\left(\vec{\lambda}^{\prime}\right)\right)}{a a^{\prime}}\right| \leq \frac{a\left|a^{\prime}-a\right|}{a a^{\prime}}+\frac{a\left|a-a^{\prime}\right|}{a a^{\prime}}=2 \frac{\left|a-a^{\prime}\right|}{a^{\prime}} \leq 4 \delta,
\end{aligned}
$$

thus implying that $\left|d_{(\vec{\lambda}, \vec{\beta})}\left(\ell_{(X, \vec{\psi})}, \ell_{(Y, \vec{\psi})}\right)-d_{\left(\vec{\lambda}, \overrightarrow{\beta^{\prime}}\right)}\left(\ell_{(X, \vec{\varphi})}, \ell_{(Y, \vec{\psi})}\right)\right| \leq 8 \delta C$, satisfying the claim since $8 \delta C \leq \delta(16 C+2)$.

Similarly, we can show that, if $\mu(\vec{\lambda})=1-a$ and $\mu\left(\vec{\lambda}^{\prime}\right)=a^{\prime}$, then $\left|d_{(\vec{\lambda}, \vec{\beta})}\left(\ell_{(X, \vec{\varphi})}, \ell_{(Y, \vec{\psi})}\right)-d_{\left(\vec{\lambda}, \vec{\beta}^{\prime}\right)}\left(\ell_{(X, \vec{\varphi})}, \ell_{(Y, \vec{\psi})}\right)\right| \leq 8 \delta C$.

If $\mu(\vec{\lambda})=1-a$ and $\mu\left(\vec{\lambda}^{\prime}\right)=1-a^{\prime}$, then it holds that $a, a^{\prime} \geq \frac{1}{2}$. Moreover, $\frac{\mu(\vec{\lambda})}{a}=\frac{1-a}{a}, \frac{\mu\left(\vec{\lambda}^{\prime}\right)}{a^{\prime}}=\frac{1-a^{\prime}}{a^{\prime}}$ and hence $\left|\frac{\mu(\vec{\lambda})}{a}-\frac{\mu\left(\overrightarrow{\vec{\lambda}^{\prime}}\right)}{a^{\prime}}\right|=\frac{\left|a^{\prime}-a\right|}{a a^{\prime}} \leq 4 \delta$, leading to the inequality $\left|d_{(\vec{\lambda}, \vec{p})}\left(\ell_{(X, \vec{\psi})}, \ell_{(Y, \vec{\psi})}\right)-d_{\left(\overrightarrow{\left.\vec{x}^{\prime}, \vec{\beta}^{\prime}\right)}\right.}\left(\ell_{(X, \vec{\varphi})}, \ell_{(Y, \vec{\psi})}\right)\right| \leq 8 \delta C$, which satisfies the claim since $8 \delta C \leq \delta(16 C+2)$. Thus, when $b, b^{\prime} \leq-C$ our statement remains proved.

Similar arguments can be used to prove our statement if $b, b^{\prime} \geq C$.

To conclude the proof we need to consider the case $|b|,\left|b^{\prime}\right| \geq C$ with $b b^{\prime}<0$. Since $\left\|(\vec{\lambda}, \vec{\beta})-\left(\vec{\lambda}^{\prime}, \vec{\beta}^{\prime}\right)\right\|_{\infty} \leq \delta$, it follows that $C \leq \frac{\delta}{2}$. Moreover, the Matching Stability Theorem 2.2 implies that $d_{\text {match }}\left(\ell_{\left(X, \varphi_{i}\right)}, \ell_{\left(Y, \psi_{i}\right)}\right) \leq 2 C$ for $i=1,2$. In the light of these considerations, we can then write

$$
\begin{aligned}
\left|d_{(\vec{\lambda}, \vec{\beta})}\left(\ell_{(X, \vec{\varphi})}, \ell_{(Y, \vec{\psi})}\right)-d_{\left(\vec{\lambda}^{\prime}, \vec{\beta}^{\prime}\right)}\left(\ell_{(X, \vec{\varphi})}, \ell_{(Y, \vec{\psi})}\right)\right| & \leq \max \left\{d_{(\vec{\lambda}, \vec{\beta})}\left(\ell_{(X, \vec{\varphi})}, \ell_{(Y, \vec{\psi})}\right), d_{\left(\vec{\lambda}^{\prime}, \vec{\beta}^{\prime}\right)}\left(\ell_{(X, \vec{\varphi})}, \ell_{(Y, \vec{\psi})}\right)\right\} \leq \\
& \leq \max \left\{d_{\text {match }}\left(\ell_{\left(X, \varphi_{1}\right)}, \ell_{\left(Y, \psi_{1}\right)}\right), d_{\text {match }}\left(\ell_{\left(X, \varphi_{2}\right)}, \ell_{\left(Y, \psi_{2}\right)}\right)\right\} \leq 2 C \leq \delta,
\end{aligned}
$$

where the first inequality in (4) is a consequence of Lemma 3.1. Clearly, (3)-(4) imply the claim, thus concluding the proof.

Remark 3.5. It is possible to prove that $d_{(\vec{\lambda}, \vec{\beta})}\left(\ell_{(X, \vec{\overrightarrow{ }})}, \ell_{(Y, \vec{\psi})}\right) \leq 2 C$ for every $(\vec{\lambda}, \vec{\beta}) \in \operatorname{Ladm} 2$ (this is a trivial consequence of [1, Thm.4]); thus $\left|d_{(\vec{\lambda}, \vec{\beta})}\left(\ell_{(X, \vec{\psi})}, \ell_{(Y, \vec{\psi})}\right)-d_{\left(\vec{\lambda}^{\prime}, \vec{\beta}^{\prime}\right)}\left(\ell_{(X, \vec{\psi})}, \ell_{(Y, \vec{\psi})}\right)\right| \leq 2 C$. Now, let us observe that if $\delta \geq \frac{1}{8}$ then $2 C \leq$ $\delta \cdot(16 C+2)$. Consequently, the inequality claimed by the Error Bound Theorem 3.4 is trivial when $\delta \geq \frac{1}{8}$.

The results proved in Lemma 3.1, Lemma 3.3 and Theorem 3.4 can be exploited to develop an algorithm able to effectively approximate the 2-dimensional matching distance $D_{\text {match }}\left(\ell_{(X, \vec{\varphi})}, \ell_{(Y, \vec{\psi})}\right)$.

By Definition 2.8 it follows that, in general, a direct computation of $D_{\text {match }}\left(\ell_{(X, \vec{\varphi})}, \ell_{(Y, \vec{\psi})}\right)$ is not possible, since we should calculate the value $d_{(\vec{\lambda}, \vec{\beta})}\left(\ell_{(X, \vec{\varphi})}, \ell_{(Y, \vec{\psi})}\right)$ for an infinite number of pairs $(\vec{\lambda}, \vec{\beta})$. On the other hand, as stressed at the end of Section 2, if we choose a non-empty and finite subset $A \subseteq \operatorname{Ladm}_{2}$, and $\operatorname{substitute} \sup _{(\vec{\lambda}, \vec{\beta}) \in \operatorname{Ladm} m_{2}}$ with $\max _{(\vec{l}, \vec{b}) \in A}$ in Definition 2.8, we get a computable pseudo-distance, say $\widetilde{D}_{\text {match }}\left(\ell_{(X, \vec{\psi})}, \ell_{(Y, \vec{\psi})}\right)$, to be used in concrete applications.

If we think of $\widetilde{D}_{\text {match }}\left(\ell_{(X, \vec{\varphi})}, \ell_{(Y, \vec{\psi})}\right)$ as an approximation of $D_{\text {match }}\left(\ell_{(X, \vec{\varphi})}, \ell_{(Y, \vec{\psi})}\right)$, it is reasonable to guess that the larger the set $A \subseteq \operatorname{Ladm}_{2}$, the smaller the difference between the two values can be. On the other hand, the smaller the set $A$, the faster the computation of $\widetilde{D}_{\text {match }}$ is. Following these considerations, we implement an algorithm in order to find a set $A$ representing a compromise between these two situations. Additionally, given an arbitrary 
real value $\varepsilon>0$ as error threshold, we want $A$ depending on $\varepsilon$ in a way that the output $\widetilde{D}_{\text {match }}\left(\ell_{(X, \vec{\varphi})}, \ell_{(Y, \vec{\psi})}\right)=$ $\max _{(\vec{\lambda}, \vec{\beta}) \in A} d_{(\vec{\lambda}, \vec{p})}\left(\ell_{(X, \vec{\overrightarrow{ }})}, \ell_{(Y, \vec{\psi})}\right)$ satisfies the inequality $\left|D_{\text {match }}\left(\ell_{(X, \vec{\varphi})}, \ell_{(Y, \vec{\psi})}\right)-\widetilde{D}_{\text {match }}\left(\ell_{(X, \overrightarrow{\vec{\varphi}})}, \ell_{(Y, \vec{\psi})}\right)\right| \leq \varepsilon$

The next section describes the algorithm in detail.

\subsection{Algorithm}

First of all, let us observe that the set $\operatorname{Ladm}_{2}=\left\{(a, 1-a, b,-b) \in \mathbb{R}^{2} \times \mathbb{R}^{2}: 0<a<1\right\}$ can be identified with the subset of $\mathbb{R}^{2}$ given by $\left\{(a, b) \in \mathbb{R}^{2}: 0<a<1\right\}=(0,1) \times \mathbb{R}$. More precisely, a bijective correspondence exists between $\operatorname{Ladm}_{2}^{*}=\left\{(a, 1-a, b,-b) \in \operatorname{Ladm}_{2}:|b|<C\right\}$ and the set $(0,1) \times(-C, C)$, as well as between $L a d m_{2} \backslash \operatorname{Ladm}_{2}^{*}$ and the set $(0,1) \times(\mathbb{R} \backslash(-C, C))$. Therefore, the finite set $A$ we are looking for can be associated with a finite subset of $(0,1) \times \mathbb{R}$. The set $A$ can be computed as follows.

1. Start by fixing the error threshold $\varepsilon$, and setting $\delta=\frac{1}{16}$ (cf. Remark 3.5);

2. Initialize $\mathcal{P}=\left\{P_{n}\right\}$, where the points $P_{n}$ define a finite and regular (square) grid in $(0,1) \times \mathbb{R}$. Choose the set $\mathcal{P}$ in a way that, taking the square centered in $P_{n}$ with side equal to $2 \delta$, i.e. $Q_{\delta}\left(P_{n}\right)=\left\{P \in \mathbb{R}^{2}:\left\|P_{n}-P\right\|_{\infty} \leq \delta\right\}$, the collection $Q=\left\{Q_{\delta}\left(P_{n}\right)\right\}$ covers the set $(0,1) \times(-C, C)$ (see Figure 3 and the following pseudo-code for details about the sets $\mathcal{P}$ and $Q$ ). Under these assumptions, and due to the bijective correspondence existing between $(0,1) \times \mathbb{R}$ and $\operatorname{Ladm}_{2}$, by Remark 3.5 and Theorem 3.4 we can control the variation of the values of $d_{(\vec{\lambda}, \vec{p})}\left(\ell_{(X, \vec{q})}, \ell_{(Y, \vec{\psi})}\right)$ associated to the points in each set $Q_{\delta}\left(P_{n}\right)$, and hence in $L a d m_{2}^{*}$;

3. Take the two points $P_{-}=\left(\frac{1}{2},-(C+1)\right), P_{+}=\left(\frac{1}{2}, C+1\right) \in(0,1) \times(\mathbb{R} \backslash(-C, C))$. Lemma 3.1 allows us to compute the maximum of $d_{(\vec{\lambda}, \vec{\beta})}\left(\ell_{(X, \vec{\varphi})}, \ell_{(Y, \vec{\psi})}\right)$ over $L a d m_{2} \backslash \operatorname{Ladm}_{2}^{*}$ just by considering the values $d_{\left(\vec{\lambda}_{-}, \vec{\beta}_{-}\right)}\left(\ell_{(X, \vec{\varphi})}, \ell_{(Y, \vec{\psi})}\right)$ and $d_{\left(\vec{\lambda}_{+}, \vec{\beta}_{+}\right)}\left(\ell_{(X, \vec{\varphi})}, \ell_{(Y, \vec{\psi})}\right)$, with $\left(\vec{\lambda}_{-}, \vec{\beta}_{-}\right)=\left(\frac{1}{2}, \frac{1}{2},-(C+1), C+1\right)$ and $\left(\vec{\lambda}_{+}, \vec{\beta}_{+}\right)=\left(\frac{1}{2}, \frac{1}{2}, C+1,-(C+1)\right)$. By Remark 3.2, this is equivalent to compute $d_{\text {match }}\left(\ell_{\left(X, \varphi_{1}\right)}, \ell_{\left(Y, \psi_{1}\right)}\right)$ and $d_{\text {match }}\left(\ell_{\left(X, \varphi_{2}\right)}, \ell_{\left(Y, \psi_{2}\right)}\right)$;

4. For every $P_{n}=\left(a_{n}, b_{n}\right) \in \mathcal{P}$, consider the associated pair $\left(\vec{\lambda}_{n}, \vec{\beta}_{n}\right)=\left(a_{n}, 1-a_{n}, b_{n},-b_{n}\right) \in \operatorname{Ladm}_{2}$, and compute the value $d_{\left(\vec{\lambda}_{n}, \vec{\beta}_{n}\right)}\left(\ell_{(X, \vec{\varphi})}, \ell_{(Y, \vec{\psi})}\right)$;

5. Compute the maximum between $\max _{P_{n} \in \mathcal{P}}\left\{d_{\left(\overrightarrow{(}_{n}, \vec{\beta}_{n}\right)}\left(\ell_{(X, \vec{\varphi})}, \ell_{(Y, \vec{\psi})}\right)\right\}, d_{\text {match }}\left(\ell_{\left(X, \varphi_{1}\right)}, \ell_{\left(Y, \psi_{1}\right)}\right)$ and $d_{\text {match }}\left(\ell_{\left(X, \varphi_{2}\right)}, \ell_{\left(Y, \psi_{2}\right)}\right)$ to obtain a first approximation of $D_{\text {match }}\left(\ell_{(X, \vec{\varphi})}, \ell_{(Y, \vec{\psi})}\right)$, say $\bar{D}$. Now,

(a) If the inequality $\delta \cdot(16 C+2) \leq \varepsilon$ holds, by Definition 2.8 and by applying Theorem 3.4 it follows that $\left|D_{\text {match }}\left(\ell_{(X, \vec{\psi})}, \ell_{(Y, \vec{\psi})}\right)-\bar{D}\right| \leq \varepsilon$. Therefore the algorithm ends, giving as output $\widetilde{D}_{\text {match }}\left(\ell_{(X, \vec{\varphi})}, \ell_{(Y, \vec{\psi})}\right)=\bar{D}$. Clearly, the set $A$ considered for the computation of $\widetilde{D}_{\text {match }}\left(\ell_{(X, \vec{\varphi})}, \ell_{(Y, \vec{\psi})}\right)$ is given by the finite set of the linearly admissible pairs associated with $\mathcal{P} \cup\left\{P_{-}, P_{+}\right\}$;

(b) Otherwise, the algorithm deletes each point $P_{n} \in \mathcal{P}$ such that $\bar{D}-d_{\left(\vec{\lambda}_{n}, \vec{\beta}_{n}\right)}\left(\ell_{(X, \vec{\varphi})}, \ell_{(Y, \vec{\psi})}\right)>\delta \cdot(16 C+2)$, and the associated set $Q_{\delta}\left(P_{n}\right) \in Q$. Indeed, Theorem 3.4 ensures that $\bar{D}$ will not be achieved (or exceeded) by computing the values $d_{(\vec{\lambda}, \vec{\beta})}\left(\ell_{(X, \vec{\varphi})}, \ell_{(Y, \vec{\psi})}\right)$ over these sets. Moreover, each $Q_{\delta}\left(P_{n}\right)$ still in $Q$ is split into four sets, and each point $P_{n}$ is substituted with the four points $P_{n++}, P_{n+-}, P_{n-+}, P_{n--}$ as shown in Figure 
4 (see the pseudo-code in Listing 1 for details about this procedure). Finally, $\delta$ is replaced by $\frac{\delta}{2}$, and the algorithm restarts from step 4 .

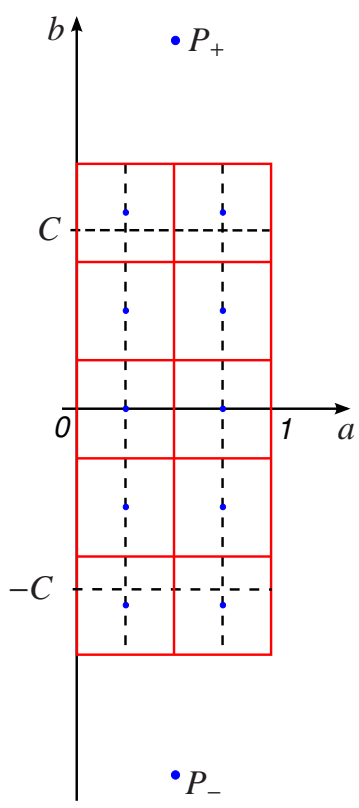

Figure 3: The starting covering for the set $(0,1) \times(-C, C)$ described in the algorithm at step 2 .

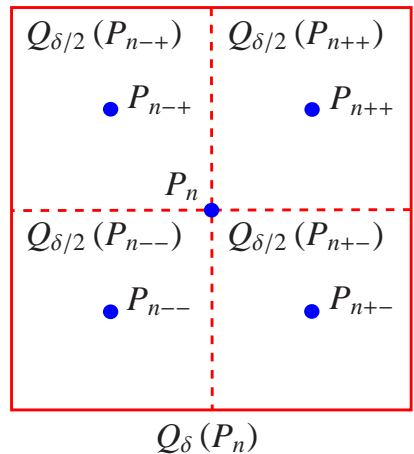

Figure 4: An application of the procedure described at step 5(b). The set $Q_{\delta}\left(P_{n}\right)$ is split into the four sets $Q_{\delta / 2}\left(P_{n-+}\right), Q_{\delta / 2}\left(P_{n++}\right), Q_{\delta / 2}\left(P_{n+-}\right)$, $Q_{\delta / 2}\left(P_{n--}\right)$, while the point $P_{n}$ is substituted with $P_{n-+}, P_{n++}, P_{n+-}, P_{n--}$.

\subsection{Computational complexity}

As it can be seen in Listing 1, the operations involved in the computation of the 2-dimensional matching distance between two models $X$ and $Y$ are the computation of the 1-dimensional size functions of $X$ and $Y$ for each point in the set $\mathcal{P}$, and their corresponding 1-dimensional matching distances. The cost of computing a 1-dimensional size 
function on a mesh with $m$ vertices takes $O(m \log m)$. Computing the 1-dimensional matching distance between two 1-dimensional size functions takes $O\left(p^{2.5}\right)$, being $p$ the total number of cornerpoints of the two descriptors [3]. Hence, the overall computational complexity depends on the complexities above, multiplied by the number of points in $\mathcal{P}$. The worst case cardinality of the set $\mathcal{P}$, that is without any point cancellation, is $O\left(\frac{C}{2 \delta^{2}}\right)$ that corresponds to cover the set $(-C, C) \times(0,1)$ with squares of side $2 \delta$. Since $\varepsilon=\delta(16 C+2)$ we obtain $O\left(\frac{C}{2 \delta^{2}}\right)=O\left(\frac{2 C(8 C+1)^{2}}{\varepsilon^{2}}\right)$. Denoting by $\bar{C}$ the constant $2 C(8 C+1)$, the number of point in $\mathcal{P}$ is $O\left(\frac{\bar{C}}{\varepsilon^{2}}\right)$. Moreover, we can estimate that the number of iterations of the algorithm is $\log _{2} \frac{8 C+1}{2 \varepsilon}$. At any rate, in our experiments this number is considerably lower (up to $4 \%$ of the worst case estimate) thanks to our cancellation strategy, see Section 4.

\section{Experiments}

We present some experiments on 3D surface mesh models taken from the SHREC 2007 database [20]. In these experiments, the 2-dimensional measuring function is $\vec{\varphi}=\left(\varphi_{1}, \varphi_{2}\right)$, with $\varphi_{1}$ the integral geodesic distance [21] and $\varphi_{2}$ the distance from the principal vector defined in [1, Section 6.2]. The values of $\vec{\varphi}$ are normalized so that they range in the interval $[0,1]$. This implies that the constant $C$ is equal to 1.

We fix the error threshold $\varepsilon$ equal to $5 \%$ of the constant $C$, that is, $\varepsilon=0.05$. Setting $\delta=\frac{1}{16}$, six iterations are required to the value $t=\delta \cdot(16 C+2)$ to become smaller than $\varepsilon$.

Figures 5, 6, 7, 8, 9 show some results, obtained by computing the approximation of the 2-dimensional matching distance on five pairs of 3D models. Each plot shows the values of $d_{(\vec{\lambda}, \vec{\beta})}\left(\ell_{(X, \vec{\varphi})}, \ell_{(Y, \vec{\psi})}\right)$ outside and inside $L a d m_{2}^{*}$. In the color coding, red corresponds to higher values, whereas blue corresponds to lower values. Outside $L a d m_{2}^{*}$, following step 3 of our algorithm, it is sufficient to compute $d_{\text {match }}\left(\ell_{\left(X, \varphi_{1}\right)}, \ell_{\left(X, \psi_{1}\right)}\right)$ and $d_{\text {match }}\left(\ell_{\left(X, \varphi_{2}\right)}, \ell_{\left(X, \psi_{2}\right)}\right)$, corresponding to the values of $d_{(\vec{\lambda}, \vec{\beta})}\left(\ell_{(X, \vec{\varphi})}, \ell_{(Y, \vec{\psi})}\right)$ at the two points $P_{-}$and $P_{+}$. respectively. Inside $L a d m_{2}^{*}$, we use the procedure described at step 5 of the algorithm to reduce computational costs and obtain an evaluation of $\max _{(\vec{\lambda}, \vec{\beta}) \in \operatorname{Ladm}_{2}^{*}} d_{(\vec{\lambda}, \vec{\beta})}\left(\ell_{(X, \vec{\varphi})}, \ell_{(Y, \vec{\psi})}\right)$ up to the chosen error threshold $\varepsilon$. The approximated value of the 2-dimensional matching distance is then the greatest of the considered values.

If the computation were done using a single tiling strategy of $L a d m_{2}^{*}$, without the point cancellation procedure introduced above, a total amount of 240284 computations for $d_{(\vec{\lambda}, \vec{\beta})}\left(\ell_{(X, \vec{\varphi})}, \ell_{(Y, \vec{\psi})}\right)$ would be required. We show in the examples that the number of computations actually needed is much lower, up to $4 \%$ of the original number. The drop in the number of computations is more evident when comparing objects belonging to different categories (Figures 5, $6,7)$ than when same-class objects are compared (Figures 8,9).

\section{Discussion and Future work}

In this paper we presented a new framework to compute an approximation of the matching distance between 2dimensional size functions. More precisely, some new theoretical results have been introduced, in order to bound the computational error in evaluating the 2-dimensional matching distance. These results lead to the definition of a new 


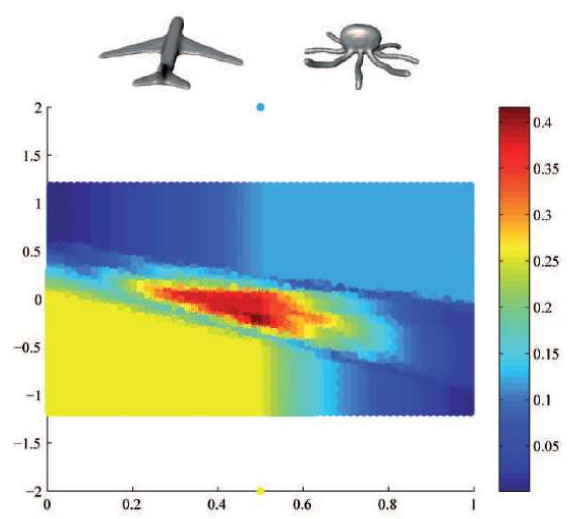

Figure 5: Comparison of an airplane model and an octopus one. The values of $d_{(\vec{\lambda}, \vec{\beta})}\left(\ell_{(X, \vec{\varphi})}, \ell_{(Y, \vec{\psi})}\right)$ at points $P_{+}$and $P_{-}$are equal to 0.178103 and 0.244962 , respectively. After 6 iterations we get 0.460318 as the maximum value taken by $d_{(\vec{\lambda}, \vec{\beta})}\left(\ell_{(X, \vec{\varphi})}, \ell_{(Y, \vec{\psi})}\right)$ in $\operatorname{Ladm}_{2}^{*}$. The approximated 2-dimensional matching distance is given by the greatest of the previous three values and is obtained by considering 13080 computations, about the $5 \%$ of those needed without the cancellation strategy.

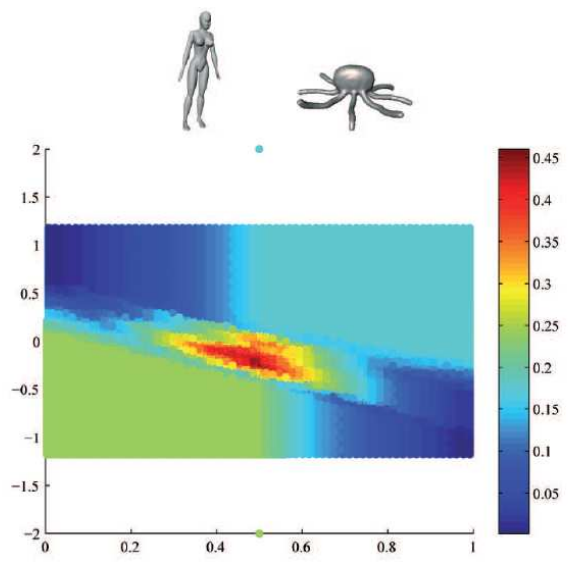

Figure 6: Comparison of a human model and an octopus one. $d_{(\vec{\lambda}, \vec{\beta})}\left(\ell_{(X, \vec{\varphi})}, \ell_{(Y, \vec{\psi})}\right)$ at points $P_{+}$and $P_{-}$is equal to 0.118791 and 0.257325 . In $L a d m_{2}^{*}$, after 6 iterations we get 0.416100 as the maximum value taken by $d_{(\vec{\lambda}, \vec{\beta})}\left(\ell_{(X, \vec{\varphi})}, \ell_{(Y, \vec{\psi})}\right)$. This value is obtained by considering 13588 computations, thus reducing of the $94 \%$ the number of operations. 


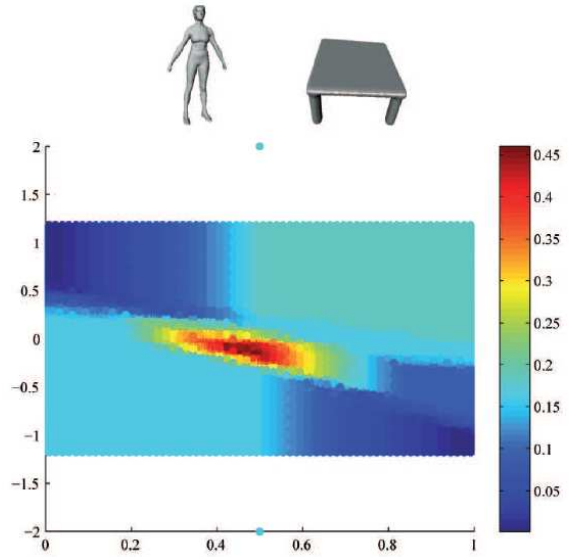

Figure 7: Comparison of a human model and a table one. $d_{(\vec{\lambda}, \vec{\beta})}\left(\ell_{(X, \vec{\varphi})}, \ell_{(Y, \vec{\psi})}\right)$ at points $P_{+}$and $P_{-}$is equal to 0.187161 and 0.159492 . Inside $L a d m_{2}^{*}$, after 6 iterations we get 0.459919 as the maximum value taken by $d_{(\vec{\lambda}, \vec{\beta})}\left(\ell_{(X, \vec{\varphi})}, \ell_{(Y, \vec{\psi})}\right)$. This value is obtained by considering 9480 computations, thus reducing of the $96 \%$ the evaluations done.

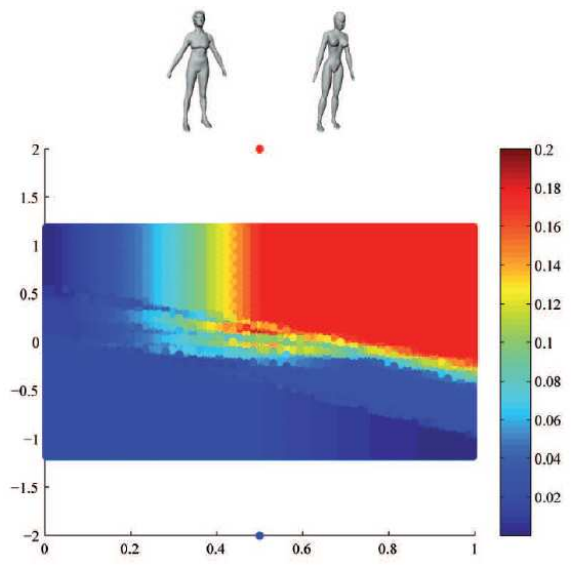

Figure 8: Comparison of two human models. $d_{(\vec{\lambda}, \vec{\beta})}\left(\ell_{(X, \vec{\varphi})}, \ell_{(Y, \vec{\psi})}\right)$ at points $P_{+}$and $P_{-}$is equal to 0.176755 and 0.019991 . In $\operatorname{Ladm}_{2}^{*}$, after 6 iterations we get 0.200139 as the maximum value taken by $d_{(\vec{\lambda}, \vec{\beta})}\left(\ell_{(X, \vec{\varphi})}, \ell_{(Y, \vec{\psi})}\right)$. This value is obtained by considering 88456 computations; i.e. the $37 \%$ of the those needed without cancellation strategy. 


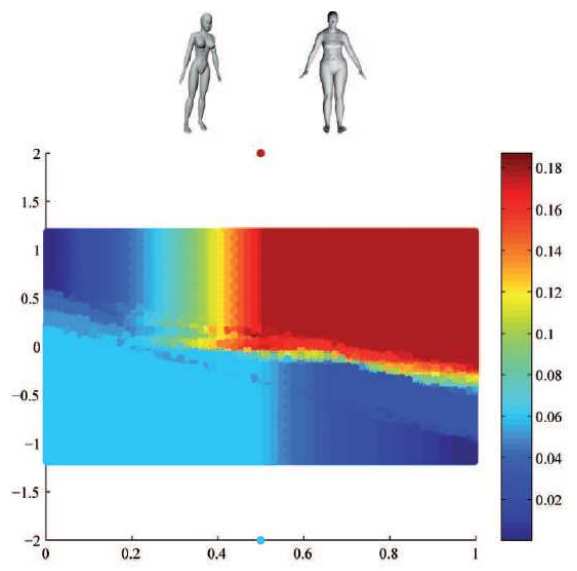

Figure 9: Comparison of two human models. $d_{(\vec{\lambda}, \vec{\beta})}\left(\ell_{(X, \vec{\varphi})}, \ell_{(Y, \vec{\psi})}\right)$ at points $P_{+}$and $P_{-}$is equal to 0.176755 and 0.060072 . Inside $L a d m_{2}^{*}$, after 6 iterations we get 0.186972 as the maximum value taken by $d_{(\vec{\lambda}, \vec{\beta})}\left(\ell_{(X, \vec{\varphi})}, \ell_{(Y, \vec{\psi})}\right)$. This value is obtained by considering 109536 computations, i.e. the $45 \%$ of those without cancellations.

algorithm to compute an approximation of the matching distance between 2-dimensional size functions in the discrete case. Our algorithm takes as input an arbitrary error threshold, representing the maximum error we are disposed to accept, giving as output an approximation of the 2-dimensional matching distance up to the chosen error threshold. The algorithm can be used in Computer Vision and Computer Graphics to compare digital shapes, as shown by some examples on 3D surface models represented by triangle meshes. It has to be noted that the definition of $k$-dimensional size functions and their matching distance holds for any $k$ [1]. Whereas in this paper we have found a computational solution for the case $k=2$, we are currently studying how to extend the algorithm to higher dimensions.

\section{Acknowledgments}

This work is carried out under the auspices of INdAM-GNSAGA, and partially supported by the CNR activities DG.RSTL.050.008 and ICT.P10.009.001.

\section{Appendix A: Proof of Lemma 3.3}

This appendix is devoted to the proof of Lemma 3.3. To this aim, we first need to introduce the following

Lemma A.1. For every $u_{1}, u_{2}, v_{1}, v_{2} \in \mathbb{R}$ it holds that

$$
\left|\max \left(u_{1}, u_{2}\right)-\max \left(v_{1}, v_{2}\right)\right| \leq \max \left(\left|u_{1}-v_{1}\right|,\left|u_{2}-v_{2}\right|\right) .
$$

Proof. Without loss of generality, we can assume that $\max \left(u_{1}, u_{2}\right)=u_{1}$. If $\max \left(v_{1}, v_{2}\right)=v_{1}$ the claim trivially follows. It only remains to check the case $\max \left(v_{1}, v_{2}\right)=v_{2}$. We have that

$$
\max \left(u_{1}, u_{2}\right)-\max \left(v_{1}, v_{2}\right)=u_{1}-v_{2} \leq u_{1}-v_{1} \leq\left|u_{1}-v_{1}\right| \leq \max \left(\left|u_{1}-v_{1}\right|,\left|u_{2}-v_{2}\right|\right) .
$$


In the same way, we obtain $\max \left(v_{1}, v_{2}\right)-\max \left(u_{1}, u_{2}\right)=v_{2}-u_{1} \leq \max \left(\left|u_{1}-v_{1}\right|,\left|u_{2}-v_{2}\right|\right)$, thus proving that $\left|\max \left(u_{1}, u_{2}\right)-\max \left(v_{1}, v_{2}\right)\right| \leq \max \left(\left|u_{1}-v_{1}\right|,\left|u_{2}-v_{2}\right|\right)$.

We are now ready to prove Lemma 3.3.

Proof of Lemma 3.3. Let $(\vec{\lambda}, \vec{\beta})=(a, 1-a, b,-b)$ and $\left(\vec{\lambda}^{\prime}, \vec{\beta}^{\prime}\right)=\left(a^{\prime}, 1-a^{\prime}, b^{\prime},-b^{\prime}\right)$. From the definition of $d_{m a t c h}$ (see also [8, Proposition 2.2]), we can write $d_{(\vec{\lambda}, \vec{\beta})}\left(\ell_{(X, \vec{\varphi})}, \ell_{(Y, \vec{\psi})}\right)=d_{\text {match }}\left(\ell_{\left(X, \mu(\vec{\lambda}) \cdot F_{(\vec{\lambda}, \vec{\beta})}^{\vec{\theta}}\right)}, \ell_{\left(Y, \mu(\vec{\lambda}) \cdot F_{(\vec{\lambda}, \vec{\beta})}^{\vec{\psi}}\right)}\right)$ for every considered $(\vec{\lambda}, \vec{\beta})$. Therefore it holds that

$$
\begin{aligned}
& \left|d_{(\vec{\lambda}, \vec{\beta})}\left(\ell_{(X, \vec{\varphi})}, \ell_{(Y, \vec{\psi})}\right)-d_{\left(\vec{\lambda}^{\prime}, \vec{\beta}^{\prime}\right)}\left(\ell_{(X, \vec{\varphi})}, \ell_{(Y, \vec{\psi})}\right)\right|= \\
& =\left|d_{\text {match }}\left(\ell_{\left(X, \mu(\vec{\lambda}) \cdot F_{(\vec{\lambda} \vec{\beta})}^{\vec{\phi}}\right)}, \ell_{\left(Y, \mu(\vec{\lambda}) \cdot F_{(\vec{\lambda}, \vec{\beta})}^{\vec{\psi}}\right)}\right)-d_{\text {match }}\left(\ell_{\left(X, \mu\left(\vec{\lambda}^{\prime}\right) \cdot F_{\left(\vec{\lambda} \vec{\lambda}^{\prime}, \vec{\beta}^{\prime}\right)}\right)}, \ell_{\left(Y, \mu\left(\vec{\lambda}^{\prime}\right) \cdot F_{\left(\vec{\lambda}^{\prime}, \vec{\beta}^{\prime}\right)}^{\vec{\psi}}\right)}\right)\right| \leq \\
& \leq d_{\text {match }}\left(\ell_{\left(X, \mu(\vec{\lambda}) \cdot F_{(\vec{\lambda} \vec{\beta})}^{\vec{\beta}}\right)}, \ell_{\left(X, \mu\left(\vec{\lambda}^{\prime}\right) \cdot F_{\left(\vec{\lambda}^{\prime}, \vec{\beta}^{\prime}\right)}^{\vec{\beta}^{\prime}}\right)}\right)+d_{\text {match }}\left(\ell_{\left(Y, \mu(\vec{\lambda}) \cdot F_{(\vec{\lambda}, \vec{\beta})}^{\vec{\psi}}\right)}, \ell_{\left(Y, \mu\left(\vec{\lambda}^{\prime}\right) \cdot F_{\left(\vec{\lambda}^{\prime}, \vec{\beta}^{\prime}\right)}^{\vec{\psi}}\right)}\right),
\end{aligned}
$$

where the last inequality comes from a trivial extension of the triangular inequality to the case of four elements. In this way we can apply the Matching Stability Theorem 2.2 to get a bound for each term in (4). Indeed, we obtain

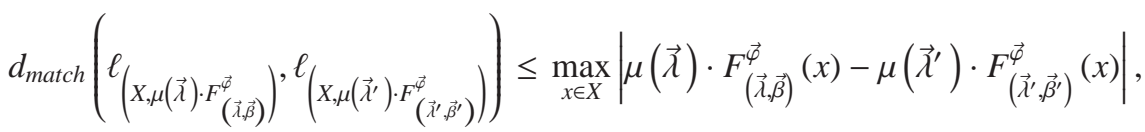

$$
\begin{aligned}
& d_{\text {match }}\left(\ell_{\left(Y, \mu(\vec{\lambda}) \cdot F_{(\vec{\lambda}, \vec{\beta})}^{\vec{\psi}}\right)}, \ell_{\left(Y, \mu\left(\vec{\lambda}^{\prime}\right) \cdot F_{\left(\vec{\prime}^{\prime}, \vec{\beta}^{\prime}\right)}^{\vec{\psi}}\right)}\right) \leq \max _{y \in Y}\left|\mu(\vec{\lambda}) \cdot F_{(\vec{\lambda}, \vec{\beta})}^{\vec{\psi}}(y)-\mu\left(\vec{\lambda}^{\prime}\right) \cdot F_{\left(\vec{\lambda}^{\prime}, \vec{\beta}^{\prime}\right)}^{\vec{\psi}}(y)\right| .
\end{aligned}
$$

By Lemma A.1, and from the definition of $F_{(\vec{\lambda}, \vec{\beta})}^{\vec{\psi}}$ and $F_{(\vec{\lambda}, \vec{\beta})}^{\vec{\psi}}$ (cf. the Reduction Theorem 2.7), in (5) and (6) we can write respectively

$$
\begin{aligned}
& \max _{x \in X}\left|\mu(\vec{\lambda}) \cdot F_{(\vec{\lambda}, \vec{\beta})}^{\vec{\varphi}}(x)-\mu\left(\vec{\lambda}^{\prime}\right) \cdot F_{\left(\vec{\lambda}^{\prime}, \vec{\beta}^{\prime}\right)}^{\vec{\varphi}}(x)\right| \leq \\
\leq & \max _{x \in X} \max \left\{\left|\mu(\vec{\lambda}) \cdot \frac{\varphi_{1}(x)-b}{a}-\mu\left(\vec{\lambda}^{\prime}\right) \cdot \frac{\varphi_{1}(x)-b^{\prime}}{a^{\prime}}\right|,\left|\mu(\vec{\lambda}) \cdot \frac{\varphi_{2}(x)+b}{1-a}-\mu\left(\vec{\lambda}^{\prime}\right) \cdot \frac{\varphi_{2}(x)+b^{\prime}}{1-a^{\prime}}\right|\right\}, \\
& \max _{y \in Y}\left|\mu(\vec{\lambda}) \cdot F_{(\vec{\lambda}, \vec{\beta})}^{\vec{\psi}}(y)-\mu\left(\vec{\lambda}^{\prime}\right) \cdot F_{\left(\vec{\lambda}^{\prime}, \vec{\beta}^{\prime}\right)}^{\vec{\psi}}(y)\right| \leq \\
\leq & \max _{y \in Y} \max \left\{\left|\mu(\vec{\lambda}) \cdot \frac{\psi_{1}(y)-b}{a}-\mu\left(\vec{\lambda}^{\prime}\right) \cdot \frac{\psi_{1}(y)-b^{\prime}}{a^{\prime}}\right|, \mid \mu(\vec{\lambda}) \cdot \frac{\psi_{2}(y)+b}{1-a}-\mu\left(\vec{\lambda}^{\prime}\right) \cdot \frac{\psi_{2}(y)+b^{\prime}}{\left.1-a^{\prime} \mid\right\} .}\right.
\end{aligned}
$$

Let us now distinguish three cases: $\left(\right.$ i) $\mu(\vec{\lambda})=a$ and $\mu\left(\vec{\lambda}^{\prime}\right)=a^{\prime},(i i) \mu(\vec{\lambda})=1-a$ and $\mu\left(\vec{\lambda}^{\prime}\right)=1-a^{\prime}$ and (iii) otherwise. 
(i). If $\mu(\vec{\lambda})=a$ and $\mu\left(\vec{\lambda}^{\prime}\right)=a^{\prime}$, then in (7) we have

$$
\begin{aligned}
& \max _{x \in X} \max \left\{\left|\mu(\vec{\lambda}) \cdot \frac{\varphi_{1}(x)-b}{a}-\mu\left(\vec{\lambda}^{\prime}\right) \cdot \frac{\varphi_{1}(x)-b^{\prime}}{a^{\prime}}\right|,\left|\mu(\vec{\lambda}) \cdot \frac{\varphi_{2}(x)+b}{1-a}-\mu\left(\vec{\lambda}^{\prime}\right) \cdot \frac{\varphi_{2}(x)+b^{\prime}}{1-a^{\prime}}\right|\right\}= \\
= & \max _{x \in X} \max \left\{\left|\varphi_{1}(x)-b-\varphi_{1}(x)+b^{\prime}\right|,\left|a \cdot \frac{\varphi_{2}(x)+b}{1-a}-a^{\prime} \cdot \frac{\varphi_{2}(x)+b^{\prime}}{1-a^{\prime}}\right|\right\}= \\
= & \max \left\{\left|b-b^{\prime}\right|, \max _{x \in X}\left|a \cdot \frac{\varphi_{2}(x)+b}{1-a}-a^{\prime} \cdot \frac{\varphi_{2}(x)+b}{1-a^{\prime}}+a^{\prime} \cdot \frac{\varphi_{2}(x)+b}{1-a^{\prime}}-a^{\prime} \cdot \frac{\varphi_{2}(x)+b^{\prime}}{1-a^{\prime}}\right|\right\}= \\
= & \max \left\{\left|b^{\prime}-b\right|, \max _{x \in X}\left|\left(\varphi_{2}(x)+b\right) \cdot\left(\frac{a}{1-a}-\frac{a^{\prime}}{1-a^{\prime}}\right)+\frac{a^{\prime}}{1-a^{\prime}} \cdot\left(b-b^{\prime}\right)\right|\right\} \leq \\
\leq & \max \left\{\left|b^{\prime}-b\right|, \max _{x \in X}\left|\varphi_{2}(x)+b\right| \cdot \frac{\left|a-a^{\prime}\right|}{(1-a)\left(1-a^{\prime}\right)}+\frac{a^{\prime}}{1-a^{\prime}} \cdot\left|b-b^{\prime}\right|\right\} \leq \\
\leq & \max \{\delta, 2 C \cdot \delta \cdot 4+\delta\}=\max \{\delta, \delta \cdot(8 C+1)\}=\delta \cdot(8 C+1),
\end{aligned}
$$

where the inequality in (14) holds since $1-a \geq \frac{1}{2}$ and $1-a^{\prime} \geq \frac{1}{2}$.

Analogously, in (8) we obtain

$$
\max _{y \in Y} \max \left\{\left|\mu(\vec{\lambda}) \cdot \frac{\psi_{1}(y)-b}{a}-\mu\left(\vec{\lambda}^{\prime}\right) \cdot \frac{\psi_{1}(y)-b^{\prime}}{a^{\prime}}\right|,\left|\mu(\vec{\lambda}) \cdot \frac{\psi_{2}(y)+b}{1-a}-\mu\left(\vec{\lambda}^{\prime}\right) \cdot \frac{\psi_{2}(y)+b^{\prime}}{1-a^{\prime}}\right|\right\} \leq \delta \cdot(8 C+1),
$$

and hence, when $\mu(\vec{\lambda})=a$ and $\mu\left(\vec{\lambda}^{\prime}\right)=a^{\prime}$, it follows that

$$
\left|d_{(\vec{\lambda}, \vec{\beta})}\left(\ell_{(X, \vec{\varphi})}, \ell_{(Y, \vec{\psi})}\right)-d_{\left(\vec{\lambda}^{\prime}, \vec{\beta}^{\prime}\right)}\left(\ell_{(X, \vec{\varphi})}, \ell_{(Y, \vec{\psi})}\right)\right| \leq \delta \cdot(16 C+2) .
$$

(ii). Working similarly to the previous case, when $\mu(\vec{\lambda})=1-a$ and $\mu\left(\vec{\lambda}^{\prime}\right)=1-a^{\prime}$ we are led to the inequality

$$
\left|d_{(\vec{\lambda}, \vec{\beta})}\left(\ell_{(X, \vec{\varphi})}, \ell_{(Y, \vec{\psi})}\right)-d_{\left(\vec{\lambda}^{\prime}, \vec{\beta}^{\prime}\right)}\left(\ell_{(X, \vec{\varphi})}, \ell_{(Y, \vec{\psi})}\right)\right| \leq \delta \cdot(16 C+2) .
$$

(iii). We can confine ourselves to the case when $\mu(\vec{\lambda})=a$ and $\mu\left(\vec{\lambda}^{\prime}\right)=1-a^{\prime}$. Indeed, the case when $\mu(\vec{\lambda})=1-a$ and $\mu\left(\vec{\lambda}^{\prime}\right)=a^{\prime}$ works similarly.

Let us consider the linear path in $\operatorname{Ladm}_{2}$ whose end points are $(\vec{\lambda}, \vec{\beta})$ and $\left(\vec{\lambda}^{\prime}, \vec{\beta}^{\prime}\right)$, that is, $\gamma:[0,1] \rightarrow \operatorname{Ladm}_{2}$, with $\gamma(t)=(1-t) \cdot(\vec{\lambda}, \vec{\beta})+t \cdot\left(\vec{\lambda}^{\prime}, \vec{\beta}^{\prime}\right)$. Since $\mu(\vec{\lambda})=a$ and $\mu\left(\vec{\lambda}^{\prime}\right)=1-a^{\prime}$, there exists at least one (exactly one when $\left.\vec{\lambda} \neq \vec{\lambda}^{\prime}\right)$ $t^{*} \in[0,1]$ such that $\gamma\left(t^{*}\right)=\left(1-t^{*}\right) \cdot(\vec{\lambda}, \vec{\beta})+t^{*} \cdot\left(\vec{\lambda}^{\prime}, \vec{\beta}^{\prime}\right)=\left(\vec{\lambda}^{*}, \vec{\beta}^{*}\right)$, with $\vec{\lambda}^{*}=\left(\frac{1}{2}, \frac{1}{2}\right)$. Moreover, from the assumption $\left\|(\vec{\lambda}, \vec{\beta})-\left(\vec{\lambda}^{\prime}, \vec{\beta}^{\prime}\right)\right\|_{\infty} \leq \delta$ we have $\left\|(\vec{\lambda}, \vec{\beta})-\left(\vec{\lambda}^{*}, \vec{\beta}^{*}\right)\right\|_{\infty} \leq t^{*} \cdot \delta$ and $\left\|\left(\vec{\lambda}^{\prime}, \vec{\beta}^{\prime}\right)-\left(\vec{\lambda}^{*}, \vec{\beta}^{*}\right)\right\|_{\infty} \leq\left(1-t^{*}\right) \cdot \delta$. Thus we can write

$$
\begin{aligned}
& \left|d_{(\vec{\lambda}, \vec{\beta})}\left(\ell_{(X, \vec{\varphi})}, \ell_{(Y, \vec{\psi})}\right)-d_{\left(\vec{\lambda}^{\prime}, \vec{\beta}^{\prime}\right)}\left(\ell_{(X, \vec{\varphi})}, \ell_{(Y, \vec{\psi})}\right)\right| \leq \\
\leq & \left|d_{(\vec{\lambda}, \vec{\beta})}\left(\ell_{(X, \vec{\varphi})}, \ell_{(Y, \vec{\psi})}\right)-d_{\left(\vec{\lambda}^{*}, \vec{\beta}^{*}\right)}\left(\ell_{(X, \vec{\varphi})}, \ell_{(Y, \vec{\psi})}\right)\right|+\left|d_{\left(\vec{\lambda}^{*}, \vec{\beta}^{*}\right)}\left(\ell_{(X, \vec{\varphi})}, \ell_{(Y, \vec{\psi})}\right)-d_{\left(\vec{\lambda}^{\prime}, \vec{\beta}^{\prime}\right)}\left(\ell_{(X, \vec{\varphi})}, \ell_{(Y, \vec{\psi})}\right)\right| \leq \\
\leq & t^{*} \cdot \delta \cdot(16 C+2)+\left(1-t^{*}\right) \cdot \delta \cdot(16 C+2)=\delta \cdot(16 C+2),
\end{aligned}
$$

where the inequality in (20) comes from (16) and (17).

By considering the bounds for the three cases $(i),(i i),($ iii $)$, we obtain the claim. 


\section{References}

[1] S. Biasotti, A. Cerri, P. Frosini, D. Giorgi, and C. Landi. Multidimensional size functions for shape comparison. J. Math. Imaging Vis., 32(2):161-179, 2008

[2] S. Biasotti, A. Cerri, and D. Giorgi. k-dimensional size functions for shape description and comparison. In R. Cucchiara, editor, ICIAP, pages 795-800. IEEE Computer Society, 2007.

[3] S. Biasotti, L. De Floriani, B. Falcidieno, P. Frosini, D. Giorgi, C. Landi, L. Papaleo, and M. Spagnuolo. Describing shapes by geometricaltopological properties of real functions. ACM Comput. Surv., 40(4):1-87, 2008.

[4] S. Biasotti, D. Giorgi, M. Spagnuolo, and B. Falcidieno. Size functions for comparing 3d models. Pattern Recogn., 41(9):2855-2873, 2008.

[5] G. Carlsson, G. Singh, and A. Zomorodian. Computing multidimensional persistence. In Y. Dong, D.-Z. Du, and O. H. Ibarra, editors, ISAAC, volume 5878 of Lecture Notes in Computer Science, pages 730-739. Springer, 2009.

[6] G. Carlsson and A. Zomorodian. The theory of multidimensional persistence. In J. Erickson, editor, Symposium on Computational Geometry, pages 184-193. ACM, 2007.

[7] A. Cerri, M. Ferri, and D. Giorgi. Retrieval of trademark images by means of size functions. Graph. Models, 68(5):451-471, 2006.

[8] A. Cerri and P. Frosini. Invariance properties of the multidimensional matching distance in Persistent Topology and Homology. Technical Report no. 2765, Università di Bologna, 2010. Available at http://amsacta.cib.unibo.it/2765/.

[9] D. Cohen-Steiner, H. Edelsbrunner, and J. Harer. Stability of persistence diagrams. In J. S. B. Mitchell and G. Rote, editors, Symposium on Computational Geometry, pages 263-271. ACM, 2005.

[10] D. Cohen-Steiner, H. Edelsbrunner, and D. Morozov. Vines and vineyards by updating persistence in linear time. In SCG 'O6: Proceedings of the twenty-second annual symposium on Computational geometry, pages 119-126, New York, NY, USA, 2006. ACM.

[11] M. d'Amico, P. Frosini, and C. Landi. Using matching distance in size theory: A survey. Int. J. Imag. Syst. Tech., 16(5):154-161, 2006.

[12] M. d'Amico, P. Frosini, and C. Landi. Natural pseudo-distance and optimal matching between reduced size functions. Acta. Appl. Math., 109:527-554, 2010.

[13] F. Dibos, P. Frosini, and D. Pasquignon. The use of size functions for comparison of shapes through differential invariants. J. Math. Imaging Vis., 21(2):107-118, 2004.

[14] H. Edelsbrunner and J. Harer. Persistent homology-a survey. In Surveys on discrete and computational geometry, volume 453 of Contemp. Math., pages 257-282. Amer. Math. Soc., Providence, RI, 2008.

[15] H. Edelsbrunner, D. Letscher, and A. Zomorodian. Topological persistence and simplification. Discr. Comput. Geom., 28(4):511-533, 2002.

[16] P. Frosini and C. Landi. Size theory as a topological tool for computer vision. Pattern Recogn. and Image Anal., 9:596-603, 1999.

[17] P. Frosini and C. Landi. Size functions and formal series. Appl. Algebra Engrg. Comm. Comput., 12(4):327-349, 2001.

[18] P. Frosini and C. Landi. Stability of multidimensional persistent homology with respect to domain perturbations, 2010. arXiv:1001.1078v2 [math.AT].

[19] P. Frosini and M. Mulazzani. Size homotopy groups for computation of natural size distances. Bull. of the Belg. Math. Soc., 6(3):455-464, 1999.

[20] D. Giorgi, S. Biasotti, and L. Paraboschi. Watertight models track. Technical Report no. 09, CNR - IMATI, Genova, Italy, 2007.

[21] M. Hilaga, Y. Shinagawa, T. Kohmura, and T. L. Kunii. Topology matching for fully automatic similarity estimation of 3d shapes. In SIGGRAPH '01: Proceedings of the 28th Annual Conference on Computer Graphics and Interactive Techniques, pages 203-212. ACM Press, 2001.

[22] A. Smeulders, M. Worring, S. Santini, A. Gupta, and R. Jain. Content-based image retrieval at the end of the early years. IEEE Trans. PAMI, 22(12), 2000.

[23] J. Tangelder and R. Veltkamp. A survey of content-based 3D shape retrieval methods. Multimedia Tools and Applications, 39(3):441-471, 2008.

[24] C. Uras and A. Verri. Computing size functions from edge maps. Int. J. Comput. Vision, 23(2):169-183, 1997.

[25] A. Verri, C. Uras, P. Frosini, and M. Ferri. On the use of size functions for shape analysis. Biol. Cybern., 70:99-107, 1993. 


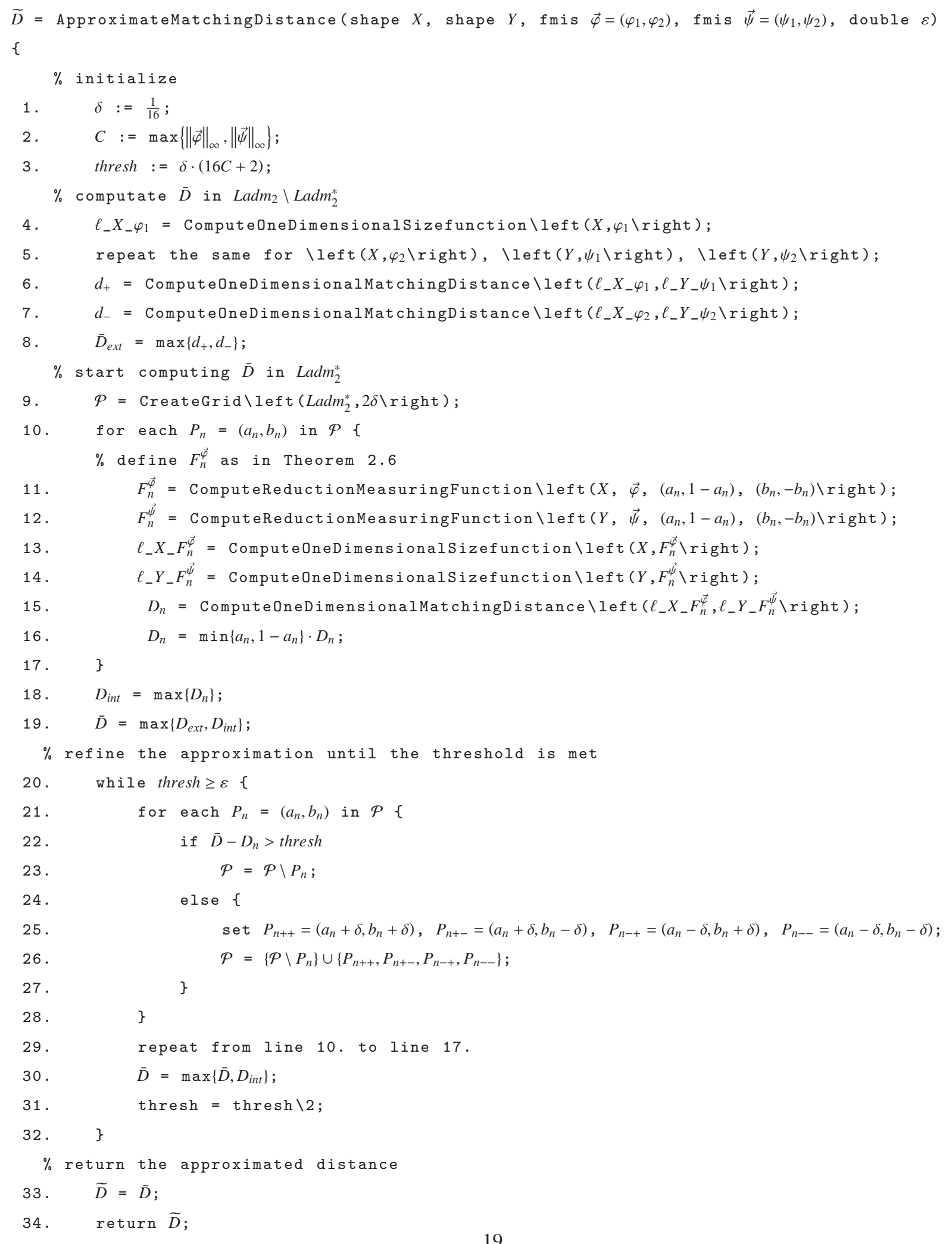

